\title{
Equimultiple locus of embedded algebroid surfaces and blowing-up in arbitrary characteristic
}

\author{
Piedra, R.* \\ Depto. de Álgebra \\ Tornero, J.M. ${ }^{\dagger}$ \\ Universidad de Sevilla \\ Depto. de Álgebra \\ Universidad de Sevilla
}

November, 2005

\begin{abstract}
This paper extends previous results of the authors, concerning the behaviour of the equimultiple locus of algebroid surfaces under blowing-up, to arbitrary characteristic.
\end{abstract}

Mathematics Subject Classification (2000): 14B05, 32S25.

\section{Introduction}

Let $K$ be an algebraically closed field of characteristic $p$ and $\mathcal{S}=$ $\operatorname{Spec}(K[[X, Y, Z]] /(F))$ an embedded algebroid surface. With no loss of generality $\mathcal{S}$ can be considered to be defined by a Weierstrass equation

$$
F(Z)=Z^{n}+\sum_{k=0}^{n-1} a_{k}(X, Y) Z^{k},
$$

where $n$ is the multiplicity of $\mathcal{S}$, that is, ord $\left(a_{k}\right) \geq n-k$ for all $k=0, \ldots, n-1$. After a change of variables we can assume that either $\bar{F}$, the initial form of $F$, is not a power of a linear form, or $\bar{F}=Z^{n}$.

*Supported by FQM 304 and BFM 2000-1523.

†Supported by FQM 218 and MTM2004-07203-C02. 
From now on, by a Weierstrass equation we will mean an equation like this.

In this situation the equimultiple locus of $\mathcal{S}$ is

$$
\mathcal{E}(\mathcal{S})=\left\{P \in \operatorname{Spec}(K[[X, Y, Z]]) \mid F \in P^{(n)}\right\},
$$

which is never empty, as $M=(X, Y, Z)$ always lies in $\mathcal{E}(\mathcal{S})$. Note that all elements of $\mathcal{E}(\mathcal{S})$, different from $M$, can be assumed to have the form $P=(Z+G(X, Y), H(X, Y))$.

Geometrically speaking, the equimultiple locus represents points at which the multiplicity is the same than in the origin; hence they are the "closest" points to the origin, in (coarse) terms of singularity complexity. We will note by $\mathcal{E}_{0}(\mathcal{S})$ the subset of smooth elements of $\mathcal{E}(\mathcal{S})$.

In our previous paper [5] we prove a theorem relating $\mathcal{E}_{0}\left(\mathcal{S}^{(1)}\right)$ to $\mathcal{E}(\mathcal{S})$, where $\mathcal{S}^{(1)}$ is the blowing-up of $\mathcal{S}$ centered in an element of $\mathcal{E}_{0}(\mathcal{S})$. Our aim is extending this result to the arbitrary characteristic case.

The main difficulty in the positive characteristic case comes from the fact that, if $p$ divides $n$, we cannot apply the Tchirnhausen transformation. This deceivingly naive procedure assures us, when it can be carried out, that:

(a) If $\bar{F}$ is the power of a linear form (that is, if the tangent cone is a plane), then it must be $\bar{F}=Z^{n}$.

(b) All the elements of $\mathcal{E}(\mathcal{S})$ contain $Z$.

(c) If, after blowing-up, the multiplicity remains the same, the above conditions still hold.

In our current situation, as we pointed out, one finds a regular parameter verifying (a) with a simple change of coordinates. Moreover, after Mulay's work ([3]) one can also find a regular parameter satisfying (b). We should note here that the outstanding result of Mulay is not constructive (much in the spirit of Abhyankar's beautiful theory on good points [1]). Even though one can manage to find a parameter verifying (a) and (b), the preservation of (a) and (b) under certain blowing-ups is false in positive characterisitic, as is well-known (see 4] for how this might be overcome in a Levi-Zariski resolution).

The existence of such an amenable parameter would have made the null characteristic proof valid for $p>0$. The peculiarities of the 
case $p \mid n$ have forced us to use completely different strategies for most parts of the result, although our proof turned to be characteristic-free, a somehow unusual fact in singularity theory.

The main result thus generalizes previous work of Zariski ([7]), Hironaka ([2]), Abhyankar ([1]) and others, who treated specific cases tailored for their purposes on resolution of surface singularities. Note that, in fact, all these results were previous to Mulay's which is a key stone in our strategy. As the geometric behaviour of the equimultiple locus of three-dimensional varieties remains unknown (see, for instance, [6]), we hope this result can be used as a first step to this much more difficult and intriguing problem.

\section{Notation and technical results}

For the sake of completeness (and for the convenience of the reader), we recall the basic facts and technical results related to quadratic and monoidal transformations that were used in [5] and which will be used afterwards.

For all what follows, let $\mathcal{S}$ be an embedded algebroid surface of multiplicity $n$,

$$
F=Z^{n}+\sum_{k=0}^{n-1}\left(\sum_{i, j} a_{i j k} X^{i} Y^{j}\right) Z^{k}=Z^{n}+\sum_{k=0}^{n-1} a_{k}(X, Y) Z^{k}
$$

a Weierstrass equation of $\mathcal{S}$. We will note

$$
N_{\{X, Y, Z\}}(F)=\left\{(i, j, k) \in \mathbf{N}^{3} \mid a_{i j k} \neq 0\right\},
$$

although we will omit the subscript whenever the variables is clear from the context.

Definition.- The elements of $\mathcal{E}(\mathcal{S})$ different from $M$ will be called equimultiple curves. The elements of $\mathcal{E}_{0}(\mathcal{S})$ other than $M$ will be called permitted curves.

Remark.- In particular, any $P \in \mathcal{E}_{0}(\mathcal{S})$ can be assumed to be, for instance $(Z, X)$, after a suitable change of variables. Clearly $(Z, X) \in$ $\mathcal{E}_{0}(\mathcal{S})$ is equivalent to $i+k \geq n$ for all $(i, j, k) \in N(F)$.

The monoidal transform of $\mathcal{S}$, centered in $(X, Z)$, in the point corresponding to the direction $(\alpha: 0: \gamma)$ (say $\alpha \neq 0$ ) of the exceptional divisor is the surface $\mathcal{S}^{(1)}$ defined by the equation

$$
F^{(1)}=\left(Z_{1}+\frac{\gamma}{\alpha}\right)^{n}+\sum_{(i, j, k) \in N(F)} a_{i j k} X_{1}^{i+k-n} Y_{1}^{j}\left(Z_{1}+\frac{\gamma}{\alpha}\right)^{k} .
$$


Observe that this only makes sense (that is, gives a non-unit) whenever $\bar{F}(\alpha, 0, \gamma)=0$. The homomorphism

$$
\begin{aligned}
\pi_{(\bar{\alpha}: 0: \gamma)}^{P}: K[[X, Y, Z]] & \longmapsto K\left[\left[X_{1}, Y_{1}, Z_{1}\right]\right] \\
X & \longmapsto X_{1} \\
Y & \longmapsto Y_{1} \\
Z & \longmapsto X_{1}\left(Z_{1}+\frac{\gamma}{\alpha}\right)
\end{aligned}
$$

will be called the homomorphism associated to the monoidal transformation in $(\bar{\alpha}: 0: \gamma)$ or, in short, the equations of the monoidal transformation. The overline is because one must privilege a non-zero coordinate, but all the possibilities define associated equations.

The quadratic transform (that is, blowing-ups with center $M$ ) in the point corresponding to the direction $(\alpha: \beta: \gamma)$ (say $\alpha \neq 0$ ) of the exceptional divisor is the surface $\mathcal{S}^{(1)}$ defined by the equation

$$
F^{(1)}=\left(Z_{1}+\frac{\gamma}{\alpha}\right)^{n}+\sum_{(i, j, k) \in N(F)} a_{i j k} X_{1}^{i+j+k-n}\left(Y_{1}+\frac{\beta}{\alpha}\right)^{j}\left(Z_{1}+\frac{\gamma}{\alpha}\right)^{k}
$$

Again this only makes sense whenever $\bar{F}(\alpha, \beta, \gamma)=0$. Analogously, the homomorphism

$$
\begin{aligned}
\pi_{(\bar{\alpha}: \beta: \gamma)}^{M}: K[[X, Y, Z]] & \longrightarrow K\left[\left[X_{1}, Y_{1}, Z_{1}\right]\right] \\
X & \longmapsto X_{1} \\
Y & \longmapsto X_{1}\left(Y_{1}+\frac{\beta}{\alpha}\right) \\
Z & \longmapsto X_{1}\left(Z_{1}+\frac{\gamma}{\alpha}\right)
\end{aligned}
$$

will be called the homomorphism associated to the quadratic transformation in $(\bar{\alpha}: \beta: \gamma)$ or the equations of the quadratic transformation. Remark.- In the previous situation, consider a change of variables in $K[[X, Y, Z]]$ given by

$$
\left\{\begin{array}{l}
\varphi(X)=a_{1} X^{\prime}+a_{2} Y^{\prime}+a_{3} Z^{\prime}+\varphi_{1}\left(X^{\prime}, Y^{\prime}, Z^{\prime}\right) \\
\varphi(Y)=b_{1} X^{\prime}+b_{2} Y^{\prime}+b_{3} Z^{\prime}+\varphi_{2}\left(X^{\prime}, Y^{\prime}, Z^{\prime}\right) \\
\varphi(Z)=c_{1} X^{\prime}+c_{2} Y^{\prime}+c_{3} Z^{\prime}+\varphi_{3}\left(X^{\prime}, Y^{\prime}, Z^{\prime}\right)
\end{array}\right.
$$

with ord $\left(\varphi_{i}\right) \geq 2$. 
Assume also that

$$
\left\{\begin{array}{l}
\alpha=a_{1} \alpha^{\prime}+a_{2} \beta^{\prime}+a_{3} \gamma^{\prime} \\
\beta=b_{1} \alpha^{\prime}+b_{2} \beta^{\prime}+b_{3} \gamma^{\prime} \\
\gamma=c_{1} \alpha^{\prime}+c_{2} \beta^{\prime}+c_{3} \gamma^{\prime}
\end{array}\right.
$$

with, say, $\gamma^{\prime} \neq 0$. Then there is a unique change of variables $\psi$ : $K\left[\left[X_{1}, Y_{1}, Z_{1}\right]\right] \longrightarrow K\left[\left[X_{1}^{\prime}, Y_{1}^{\prime}, Z_{1}^{\prime}\right]\right]$ such that

$$
\psi \pi_{(\bar{\alpha}: \beta: \gamma)}^{M}=\pi_{\left(\alpha^{\prime}: \beta^{\prime}: \overline{\gamma^{\prime}}\right)}^{M} \varphi .
$$

Definition.- Let $Q \in \mathcal{E}(\mathcal{S})$, with $Q=(Z+H(X, Y), G(X, Y))$. Then for $\underline{u} \in \mathbf{P}^{2}(K)$, the ideal

$$
\varpi_{\underline{u}}^{M}(Q)=\left(\frac{\pi_{\underline{u}}^{M}(Z+H(X, Y))}{X_{1}}, \frac{\pi_{\underline{u}}^{M}(G(X, Y))}{X_{1}^{\operatorname{Ord}(G)}}\right)
$$

is called the (strict) quadratic transform of $Q$ in the point $\underline{u}$.

Obviously, this definition makes sense only if the quadratic transform in the direction $\underline{u}$ does. There is a natural version of monoidal transform of $Q$ with center $P$, for all $P \in \mathcal{E}_{0}(\mathcal{S})$.

Notation.- We will note by $\nu$ the natural isomorphism

$$
\nu: K[[X, Y, Z]] \longrightarrow K\left[\left[X_{1}, Y_{1}, Z_{1}\right]\right]
$$

sending $X$ to $X_{1}, Y$ to $Y_{1}$ and $Z$ to $Z_{1}$.

\section{The theorem}

We will restrict ourselves to the case which is interesting for desingularization issues: that where $\mathcal{S}$ and $\mathcal{S}^{(1)}$ have the same multiplicity. This leaves out some situations.

Lemma.- If the tangent cone of $\mathcal{S}$ is not a plane, the multiplicity of any monoidal transform is strictly less than $n$.

Proof.- See [5] for a characteristic-free proof.

Remark.- In particular, note that if the tangent cone is not a plane, there cannot be more than one permitted curve. In fact, assume we have two curves, $P$ and $Q$, and choose $Z$ to be a regular parameter with $P=(Z, G(X, Y)), Q=(Z, H(X, Y))$. If $F$ is a Weierstrass equation with the usual form, then $P$ is permitted if and only if $G^{n-k} \mid a_{k}$ for all $k=0, \ldots, n-1$. As the same goes for $Q$, it is clearly impossible that 
there exists some $a_{k}$ with $\operatorname{ord}\left(a_{k}\right)=n-k$. Hence the tangent cone must be a plane (in fact it must be $Z=0$ ).

Theorem.- Let $\mathcal{S}$ be an algebroid surface and $\mathcal{S}^{(1)}$ a quadratic or monoidal transform of $\mathcal{S}$ having the same multiplicity.

(a) Let $\mathcal{S}^{(1)}$ be the monoidal transform of $\mathcal{S}$ with center $P \in \mathcal{E}_{0}(\mathcal{S})$ then, either $\mathcal{E}_{0}\left(\mathcal{S}^{(1)}\right)=\nu\left(\mathcal{E}_{0}(\mathcal{S})\right)$ or $\mathcal{E}_{0}\left(\mathcal{S}^{(1)}\right)=\nu\left(\mathcal{E}_{0}(\mathcal{S}) \backslash\{P\}\right)$.

(b) Let $\mathcal{S}^{(1)}$ be the quadratic transform of $\mathcal{S}$ in the point $\underline{u}$.

(b.1) If the tangent cone is not a plane then $\mathcal{E}_{0}\left(\mathcal{S}^{(1)}\right)=$ $\varpi_{\underline{u}}^{M}\left(\mathcal{E}_{0}(\mathcal{S})\right)$.

(b.2) If the tangent cone is a plane then we can find three types of curves in $\mathcal{E}_{0}\left(\mathcal{S}^{(1)}\right)$ :

(i) The exceptional divisor of the transform.

(ii) Primes $\varpi_{\underline{u}}^{M}(Q)$, with $Q \in \mathcal{E}(\mathcal{S}) \backslash \mathcal{E}_{0}(\mathcal{S})$, which are tangent to the exceptional divisor.

(iii) Primes $\varpi_{\underline{u}}^{M}(Q)$, with $Q \in \mathcal{E}_{0}(\mathcal{S})$, where both $\nu(Q)$ and $\varpi_{\underline{u}}^{M}(Q)$ are transversal to the exceptional divisor.

Moreover, if $E_{0}\left(\mathcal{S}^{(1)}\right)$ contains a prime of type (ii), then it also contains a prime of type (i).

Proof.- Although some partial results are common to the characteristic 0 case, we will repeat them or, at the very least, we will give a detailed outline when appropriate for the convenience of the reader. In what follows let $F$ be, as usual, a Weierstrass equation of $\mathcal{S}$.

\section{Case (a)}

This case presents the first (small) differences of argumentation with the characteristic 0 , as the reader can check with [5]. We have to prove that, after a monoidal transformation with center $P \in \mathcal{E}_{0}(\mathcal{S})$, $Q \in \mathcal{E}_{0}(\mathcal{S})$ if and only if $\nu(Q) \in \mathcal{E}_{0}\left(\mathcal{S}^{(1)}\right)$, except maybe for $Q=P$.

Hence assume $Z$ is a parameter verifying that for all $I \in \mathcal{E}(\mathcal{S})$, $Z \in I$. After a change of variables in $K[[X, Y]]$, we can assume $P$ to be $(Z, X)$ and $Q$ (other than $P$ ) to be $(Z, G(X, Y))$ with $\operatorname{ord}(G)=1$. As we noticed above $\bar{F}=Z^{n}$, hence there is only one direction in the exceptional divisor, $(1: 0: 0)$. An equation for $\mathcal{S}^{(1)}$ is then

$$
F^{(1)}=Z_{1}^{n}+\sum_{k=0}^{n-1} \frac{a_{k}\left(X_{1}, Y_{1}\right)}{X_{1}^{n-k}} Z_{1}^{k}=Z_{1}^{n}+\sum_{k=0}^{n-1} a_{k}^{(1)}\left(X_{1}, Y_{1}\right) Z_{1}^{k},
$$

and therefore $G\left(X_{1}, Y_{1}\right)^{n-k} \mid a_{k}^{(1)}\left(X_{1}, Y_{1}\right)$ if and only if $G(X, Y)^{n-k} \mid a_{k}(X, Y)$. This finishes the case. 


\section{Case (b.2)}

Much like in zero characteristic, this case gives the basis for the other one. It is also the point where the main differences between both cases become notorious.

Remark.- First of all note that we can restrict ourselves to the case where the tangent cone is $Z=0$ (this is as before an easy change of variables) and the direction in the exceptional divisor is $(1: 0: 0)$. If this is not the case, for the results at points $(1: \alpha: 0)$ it suffices considering the (commutative) diagram

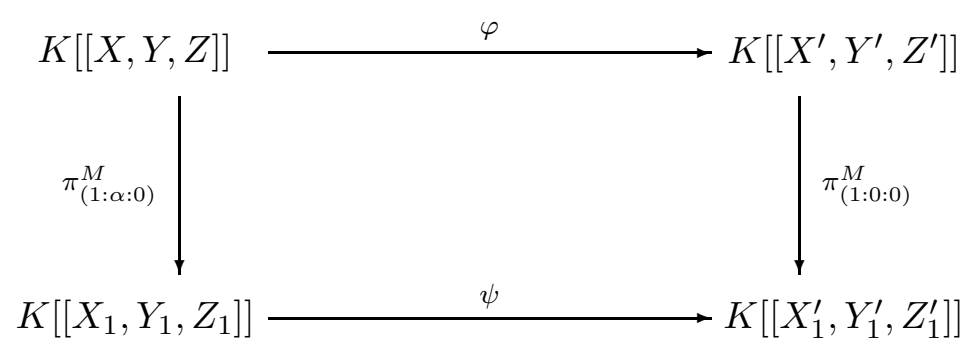

with $\varphi$ given by

$$
\left\{\begin{array}{l}
\varphi(X)=X^{\prime} \\
\varphi(Y)=Y^{\prime}+\alpha X^{\prime} \\
\varphi(Z)=Z^{\prime}
\end{array}\right.
$$

Of course the results at $(0: 1: 0)$ are clearly symmetric.

Remark.- Assume that we have a permitted curve in $\mathcal{S}^{(1)}$ of the general type, say, $Q=\left(G_{1}, G_{2}\right)$, with

$$
\begin{array}{ll}
G_{1}=\alpha_{1} X_{1}+\beta_{1} Y_{1}+\gamma_{1} Z_{1}+G_{1}^{\prime}\left(X_{1}, Y_{1}, Z_{1}\right), & \text { where ord }\left(G_{1}^{\prime}\right)>1 \\
G_{2}=\alpha_{2} X_{1}+\beta_{2} Y_{1}+\gamma_{2} Z_{1}+G_{2}^{\prime}\left(X_{1}, Y_{1}, Z_{1}\right), & \text { where ord }\left(G_{2}^{\prime}\right)>1
\end{array}
$$

As the multiplicity remains the same, the monomial $Z_{1}^{n}$ must appear in $\overline{F^{(1)}}$. Hence either $\gamma_{1}$ or $\gamma_{2}$ must be non zero. Let us suppose it is $\gamma_{1} \neq 0$ and so we can substitute $G_{1}$ by its associated Weierstrass polynomial with respect to $Z_{1}$, of the form $Z_{1}+a\left(X_{1}, Y_{1}\right)$ with $\operatorname{ord}(a) \geq 1$. Now we change $Z_{1}$ by $-a\left(X_{1}, Y_{1}\right)$ in $G_{2}$ to obtain

$$
Q=\left(Z_{1}+a\left(X_{1}, Y_{1}\right), \alpha X_{1}+\beta Y_{1}+b\left(X_{1}, Y_{1}\right)\right),
$$

with $\operatorname{ord}(a), \operatorname{ord}(b)>1$.

We will look first at permitted curves in $\mathcal{S}^{(1)}$ which are transversal to the exceptional divisor. 
Lemma.- Under the hypothesis of case (b.2) assume that there is a permitted curve $Q \in \mathcal{E}_{0}\left(\mathcal{S}^{(1)}\right)$ which is transversal to the exceptional divisor. Then there exists some $P \in \mathcal{E}_{0}(\mathcal{S})$ such that $Q=\varpi_{(1: 0: 0)}^{M}(P)$ and $\nu(P)$ is transversal to the exceptional divisor.

Proof. - In the above notation, $Q$ is transversal whenever $\beta \neq 0$. In this case we can change $\alpha X_{1}+\beta Y_{1}+b\left(X_{1}, Y_{1}\right)$ for its associated Weierstrass polynomial with respect to $Y_{1}$ and then make the corresponding substitution in $a\left(X_{1}, Y_{1}\right)$ to obtain

$$
Q=\left(Z_{1}+a^{\prime}\left(X_{1}\right), Y_{1}+b^{\prime}\left(X_{1}\right)\right)
$$

with ord $\left(a^{\prime}\right)>1$, ord $\left(b^{\prime}\right)>0$. Consider then the following diagram

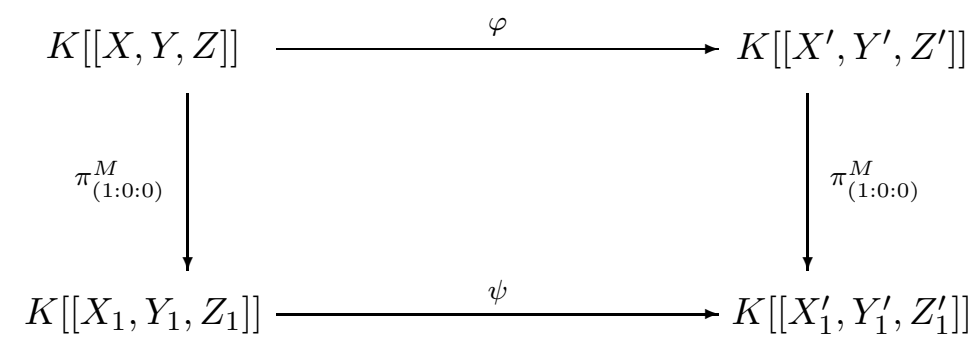

with changes of variables

$$
\left\{\begin{array}{l}
\varphi(X)=X^{\prime} \\
\varphi(Y)=Y^{\prime}-X^{\prime} b^{\prime}\left(X^{\prime}\right) \\
\varphi(Z)=Z^{\prime}-X^{\prime} a^{\prime}\left(X^{\prime}\right)
\end{array}, \quad\left\{\begin{array}{l}
\psi\left(X_{1}\right)=X_{1}^{\prime} \\
\psi\left(Y_{1}\right)=Y_{1}^{\prime}-b^{\prime}\left(X_{1}^{\prime}\right) \\
\psi\left(Z_{1}\right)=Z_{1}^{\prime}-a^{\prime}\left(X_{1}^{\prime}\right)
\end{array}\right.\right.
$$

As $\psi(Q)=\left(Z_{1}^{\prime}, Y_{1}^{\prime}\right)$, we know that $\left(Z_{1}^{\prime}, Y_{1}^{\prime}\right)$ is permitted in $\mathcal{S}^{(1)}$. But, looking at the equations of the transformation $\pi_{(1: 0: 0)}^{M}$ this clearly implies that $\left(Z^{\prime}, Y^{\prime}\right)$ was permitted in $\mathcal{S}$. Therefore

$$
P=\varphi^{-1}\left(Z^{\prime}, Y^{\prime}\right)=\left(Z+X a^{\prime}(X), Y+X b^{\prime}(X)\right)
$$

was permitted in $\mathcal{S}$. It is clear that $\nu(P)$ is transversal to the exceptional divisor and $\varpi_{(1: 0: 0)}^{M}(Q)=P$. This proves the lemma.

Now we will prove that the existence of permitted curves tangent to the exceptional divisor implies that the exceptional divisor lies in $\mathcal{E}_{0}\left(\mathcal{S}^{(1)}\right)$. We will do it without using the fact that $\bar{F}$ is the power of a linear form, and so it will be still valid for case (b.1). 
Lemma.- Under the hypothesis of case (b) assume that there is a permitted curve $P \in \mathcal{E}_{0}\left(\mathcal{S}^{(1)}\right)$ which is tangent to the exceptional divisor. Then the exceptional divisor lies in $\mathcal{E}_{0}\left(\mathcal{S}^{(1)}\right)$.

Proof.- From the remarks at the beginning of this case we can already assume that $P$ has the form

$$
P=\left(Z_{1}+a\left(Y_{1}\right), X_{1}+b\left(Y_{1}\right)\right), \text { with } \operatorname{ord}(a), \operatorname{ord}(b) \geq 2 .
$$

The proof is considerably different, depending on whether $a\left(Y_{1}\right)$ is zero or not. Let us assume $a\left(Y_{1}\right)=0$ (this is the easy part) and let us write $F^{(1)}$ as usual:

$$
F^{(1)}=Z_{1}^{n}+\sum_{k=0}^{n-1} a_{k}^{(1)}\left(X_{1}, Y_{1}\right) Z_{1}^{k}
$$

with the following decomposition

$$
a_{k}^{(1)}\left(X_{1}, Y_{1}\right)=b_{k}\left(X_{1}, Y_{1}\right)\left(X_{1}+b\left(Y_{1}\right)\right)^{n-k}, k=0, \ldots, n-1,
$$

where $b_{k}$ does not divide $X_{1}+b\left(Y_{1}\right)$.

Let us take any $k \in\{0, \ldots, n-1\}$, let $t=\operatorname{ord}(b) \geq 2$ and let us call $X_{1}^{r} Y_{1}^{s}$ the smallest monomial with respect to the lexicographic order appearing in $b_{k}\left(X_{1}, Y_{1}\right)$. Then the monomial $X_{1}^{r} Y_{1}^{s+t(n-k)} Z_{1}^{k}$ must occur in $a_{k}^{(1)}\left(X_{1}, Y_{1}\right) Z_{1}^{k}$.

Now, as this monomial appears after a quadratic transform in the point $(1: 0: 0)$ of the exceptional divisor it is clear that it must hold

$$
r \geq s+t(n-k)+k-n \geq s+2(n-k)-(n-k) \geq n-k,
$$

and therefore $X_{1}^{n-k} \mid b_{k}\left(X_{1}, Y_{1}\right)$ for all $k$. This proves that the exceptional divisor is also permitted.

Let us move now to the more complicated case, when $a\left(Y_{1}\right) \neq 0$. Now we write $F^{(1)}$ in the following form

$F^{(1)}=\left(Z_{1}+a\left(Y_{1}\right)\right)^{n}+\sum_{k=0}^{n-1} b_{k}\left(X_{1}, Y_{1}\right)\left(X_{1}+b\left(Y_{1}\right)\right)^{n-k}\left(Z_{1}+a\left(Y_{1}\right)\right)^{k}$,

for some $b_{k}\left(X_{1}, Y_{1}\right) \in K\left[\left[X_{1}, Y_{1}\right]\right]$. Hence the summand $a\left(Y_{1}\right)^{n}$, which is a power series in $K\left[\left[Y_{1}\right]\right]$ of order strictly greater than $n$, appears in the independent term.

But as $F^{(1)}$ comes from $F$ after a quadratic transformation at the point $(1: 0: 0)$ of the exceptional divisor, there can be no monomials 
in $K\left[\left[Y_{1}\right]\right]$ of order strictly greater then $n$ in the independent term, which implies clearly that $b\left(Y_{1}\right)$ cannot be 0 .

Let us write $a\left(Y_{1}\right)=Y_{1}^{r} u\left(Y_{1}\right), b\left(Y_{1}\right)=Y_{1}^{s} v\left(Y_{1}\right)$, for some $u, v$ units in $K\left[\left[Y_{1}\right]\right]$. Then, as previously, it must hold

$$
Y_{1}^{r n} u\left(Y_{1}\right)^{n}+\sum_{k=0}^{n-1} b_{k}\left(0, Y_{1}\right) Y_{1}^{r k} u\left(Y_{1}\right)^{k} Y_{1}^{s(n-k)} v\left(Y_{1}\right)^{n-k}=0
$$

which in particular implies $r n \geq s n$, that is, $r \geq s$.

Let us write $r=r^{\prime} s+t$ with $t \in\{0, \ldots, s-1\}$ and $r^{\prime} \geq 1$. Then we can rewrite our ideal as

$$
\begin{aligned}
P & =\left(Z_{1} \pm Y_{1}^{t} X_{1}^{r^{\prime}}\left(\frac{u\left(Y_{1}\right)}{v\left(Y_{1}\right)^{r^{\prime}}}\right), X_{1}+Y_{1}^{s} v\left(Y_{1}\right)\right) \\
& =\left(Z_{1}+X_{1}^{r^{\prime}} c\left(Y_{1}\right), X_{1}+Y_{1}^{s} v\left(Y_{1}\right)\right),
\end{aligned}
$$

or, alternatively

$$
P=\left(Z_{1}+X_{1} g\left(X_{1}, Y_{1}\right), X_{1}+b\left(Y_{1}\right)\right) .
$$

With this new form it must hold

$$
\begin{aligned}
F^{(1)}=( & \left.Z_{1}+X_{1} g\left(X_{1}, Y_{1}\right)\right)^{n}+ \\
& +\sum_{k=0}^{n-1} c_{k}\left(X_{1}, Y_{1}\right)\left(X_{1}+b\left(Y_{1}\right)\right)^{n-k}\left(Z_{1}+X_{1} g\left(X_{1}, Y_{1}\right)\right)^{k} .
\end{aligned}
$$

We want to prove $X_{1}^{n-k} \mid c_{k}\left(X_{1}, Y_{1}\right)$ for $k=0, \ldots, n-1$. If this were not so, let $k_{0}$ be the biggest index for which this does not happen. Let us write $X_{1}^{p} Y_{1}^{q}$ the smaller monomial for the lexicographic ordering appearing in $c_{k_{0}}\left(X_{1}, Y_{1}\right)$. Note that $p<n-k_{0}$. Then the monomials in

$$
X_{1}^{p} Y_{1}^{q} b\left(Y_{1}\right)^{n-k_{0}} Z_{1}^{k_{0}}
$$

cannot cancel with any others coming from the expansions of the remaining summands. This is so because, if $k>k_{0}$ the monomials in the $k$-th summand are in $\left(Z_{1}, X_{1}\right)^{n}$ and if $k<k_{0}$ the monomials in the $k$-th summand have smaller exponent in $Z_{1}$. Hence the monomial

$$
X_{1}^{p} Y_{1}^{q+s\left(n-k_{0}\right)} Z_{1}^{k_{0}}
$$


actually appears in $F^{(1)}$. As $F^{(1)}$ is the result of a quadratic transform in $(1: 0: 0)$, we have

$p \geq q+s\left(n-k_{0}\right)+k_{0}-n=q+(s-1)\left(n-k_{0}\right) \geq q+n-k_{0} \geq n-k_{0}$,

which contradicts our assumption. This finishes the proof of the lemma.

Note that, from the previous arguments, a curve which is tangent to the exceptional divisor can also be written as

$$
P=\left(Z_{1}+h\left(X_{1}, Y_{1}\right), X_{1}+b\left(Y_{1}\right)\right),
$$

where $b\left(Y_{1}\right) \in K\left[\left[Y_{1}\right]\right]$ and $h\left(X_{1}, Y_{1}\right) \in K\left[\left[X_{1}\right]\right]\left[Y_{1}\right]$, with $\operatorname{ord}(b)=s \geq$ 2 and $\operatorname{deg}_{Y_{1}}(h)<s$. This will be our preferred form in what follows.

In order to prove the existence of a singular equimultiple curve in $\mathcal{E}(\mathcal{S})$ which is taken into the permitted curve that is tangent to the exceptional divisor, we will begin by proving that we can find a convenient parameter to work with in this situation.

Lemma.- In the situation of case (b.2), let us assume that, after the quadratic transform in the direction $(1: 0: 0)$ of the exceptional divisor, we have the following permitted curve in $\mathcal{S}^{(1)}$ :

$$
P=\left(Z_{1}+h\left(X_{1}, Y_{1}\right), X_{1}+b\left(Y_{1}\right)\right),
$$

with $\operatorname{ord}(b)=s>1$ and $h\left(X_{1}, Y_{1}\right) \in K\left[\left[X_{1}\right]\right]\left[Y_{1}\right]$ with $\operatorname{deg}_{Y_{1}}(h)<s$.

Then there exists an isomorphism $\varphi: K[[X, Y, Z]] \longrightarrow$ $K\left[\left[X^{\prime}, Y^{\prime}, Z^{\prime}\right]\right]$ which makes the following diagram commutative

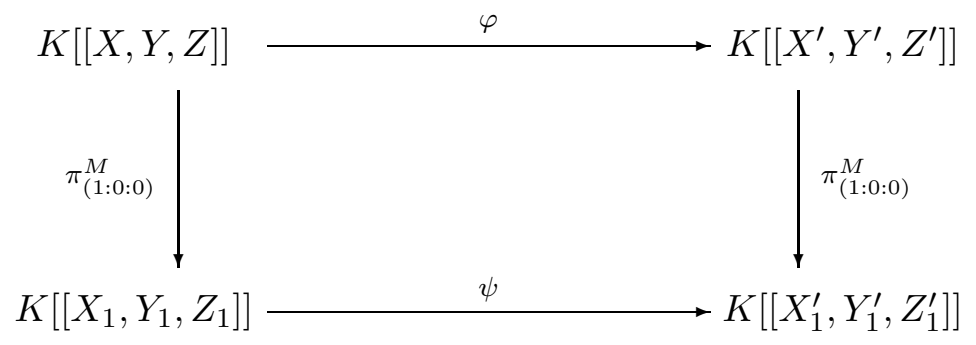

with $\psi$ given by

$$
\left\{\begin{array}{l}
\psi\left(X_{1}\right)=X_{1}^{\prime} \\
\psi\left(Y_{1}\right)=Y_{1}^{\prime} \\
\psi\left(Z_{1}\right)=Z_{1}^{\prime}-h\left(X_{1}^{\prime}, Y_{1}^{\prime}\right)
\end{array}\right.
$$


Proof.- Let us write

$$
h\left(X_{1}, Y_{1}\right)=h_{1}\left(X_{1}, Y_{1}\right)+h_{2}\left(X_{1}, Y_{1}\right),
$$

where the monomials of $h_{1}$ are precisely those $X_{1}^{\alpha} Y_{1}^{\beta}$ with $1+\alpha \geq \beta$, all the remaining ones being in $h_{2}$. Note that $h_{2} \in K\left[X_{1}, Y_{1}\right]$.

If $h_{2}=0$ then we get the result we look for with

$$
\left\{\begin{array}{l}
\varphi(X)=X^{\prime} \\
\varphi(Y)=Y^{\prime} \\
\varphi(Z)=Z^{\prime}-X^{\prime} h_{1}\left(X^{\prime}, \frac{Y^{\prime}}{X^{\prime}}\right)
\end{array}\right.
$$

If $h_{2} \neq 0$ then the previous isomorphism $\varphi$, together with

$$
\left\{\begin{array}{l}
\psi\left(X_{1}\right)=X_{1}^{\prime} \\
\psi\left(Y_{1}\right)=Y_{1}^{\prime} \\
\psi\left(Z_{1}\right)=Z_{1}^{\prime}-h_{1}\left(X_{1}^{\prime}, Y_{1}^{\prime}\right)
\end{array}\right.
$$

makes the diagram commute. In this case, we would have the permitted curve

$$
\psi(P)=\left(Z_{1}^{\prime}+h_{2}\left(X_{1}^{\prime}, Y_{1}^{\prime}\right), X_{1}^{\prime}+b\left(Y_{1}^{\prime}\right)\right)
$$

in the equimultiple locus of the surface $\mathcal{S}^{(1)}$. The equation for $\mathcal{S}^{(1)}$, in $\left\{X_{1}^{\prime}, Y_{1}^{\prime}, Z_{1}^{\prime}\right\}$ can then be written as

$$
\begin{aligned}
F^{(1)}=\left(Z_{1}^{\prime}\right. & \left.+h_{2}\left(X_{1}^{\prime}, Y_{1}^{\prime}\right)\right)^{n}+ \\
& +\sum_{k=0}^{n-1} b_{k}\left(X_{1}^{\prime}, Y_{1}^{\prime}\right)\left(X_{1}^{\prime}+b\left(Y_{1}^{\prime}\right)\right)^{n-k}\left(Z_{1}^{\prime}+h_{2}\left(X_{1}^{\prime}, Y_{1}^{\prime}\right)\right)^{k} .
\end{aligned}
$$

For the sake of simplicity, we will rename our variables as $\{X, Y, Z\}$, as no further changes of variables are needed from this point onwards.

Assume $(j, d)$ is the smallest exponent in $h_{2}(X, Y)$ for the lexicographic ordering verifying $1+j<d \leq s-1$. Let us prove

$$
X^{d+(n-k-1) j} \mid b_{k}(X, Y), \text { for all } k=0, \ldots, n-1 .
$$

SteP 1.- Let us begin by the case $k=n-1$; we want to prove $X^{d} \mid b_{n-1}(X, Y)$.

The coeffcient of $Z^{n-1}$ in $F^{(1)}$ must be

$$
\left(\begin{array}{c}
n \\
1
\end{array}\right) h_{2}+b_{n-1}(X, Y)(X+b(Y)) \text {. }
$$


Let $(\alpha, \beta)$ be the minimal exponent appearing in $b_{n-1}(X, Y)$ with respect to the lexicographic ordering. Then $(\alpha, \beta+s)$ is the minimal exponent appering in $b_{n-1}(X, Y)(X+b(Y))$ and it cannot cancel with any monomial in $h_{2}$ as $\operatorname{deg}_{Y}\left(h_{2}\right) \leq s-1$. Hence $(\alpha, \beta+s, n-1) \in$ $N\left(F^{(1)}\right)$ and, since $F^{(1)}$ is the equation for a quadratic transform in $(1: 0: 0)$, it must hold

$$
\alpha \geq \beta+s+(n-1)-n=\beta+s-1 \geq s-1 \geq d .
$$

STEP 2.- Let us assume the result is true for $k, k+1, \ldots, n-1$ and let us prove

$$
X^{d+(n-k) j} \mid b_{k-1}(X, Y) .
$$

We fix now our attention in the coefficient of $Z^{k-1}$ in $F^{(1)}$, which is formed by the expansion of

$$
b_{k-1}(X, Y)(X+b(Y))^{n-k+1},
$$

and also by monomials coming from the expression

$$
\left(Z+h_{2}\right)^{n}+(X+b) b_{n-1}\left(Z+h_{2}\right)^{n-1}+\ldots+(X+b)^{n-k} b_{k}\left(Z+h_{2}\right)^{k} .
$$

If we mimic the case above and denote by $(\alpha, \beta)$ the minimal exponent appearing in $b_{k-1}(X, Y)$ for the lexicographic ordering, then the minimal exponent for $b_{k-1}(X+b)^{n-k+1}$ must be $(\alpha, \beta+(n-k+1) s)$. And, furthermore, this exponent cannot be cancelled with any other from the developement of the summands of the expression above. Let us see this with closer detail.

Clearly, it cannot cancel with any monomial from

$$
\left(\begin{array}{c}
n \\
k-1
\end{array}\right) h_{2}(X, Y)^{n-k+1}
$$

as all of their degrees with respect to $Y$ are smaller than $(s-1)(n-$ $k+1)$.

Now, if $\alpha<d+(n-k) j$ there cannot be cancellation with monomials of

$$
\left(\begin{array}{c}
n-m \\
k-1
\end{array}\right) b_{n-m}(X+b)^{m} h_{2}^{n-m-k+1},(\text { for } m=1, \ldots, n-k),
$$

as their orders with repect to $X$ are greater or equal (by induction hypothesis) than

$$
d+(m-1) j+j(n-m-k+1)=d+j(n-k) .
$$


Hence $(\alpha, \beta+(n-k+1) s, k-1) \in N\left(F^{(1)}\right)$ and, as before, it must then hold

$$
\begin{array}{rlrl}
\alpha & \geq \beta+(n-k+1) s+k-1-n & \geq(n-k+1)(s-1) \\
& \geq(n-k+1) d & =d+(n-k) d \\
& >d+(n-k) j, & &
\end{array}
$$

which is a contradiction. Hence $X^{d+(n-k-1) j} \mid b_{k}(X, Y)$, for all $k=$ $0, \ldots, n-1$.

Now the independent term of $F^{(1)}$ is

$$
F^{(1)}(X, Y, 0)=h_{2}^{n}+\sum_{k=0}^{n-1} b_{k}(X+b)^{n-k} h_{2}^{k} .
$$

The first summand features the exponent $(n j, n d, 0)$, which must be in $N\left(F^{(1)}\right)$, as the order with respect to $X$ of any other monomial is, for some $k \in\{0, \ldots, n-1\}$ greater or equal than

$$
d+(n+k-1) j+k j=d+(n-i) j>n j+1>n j .
$$

However, this monomial cannot appear in $F^{(1)}$, as $j<d$ and $F^{(1)}$ is the equation of a quadratic transform in $(1: 0: 0)$. This shows $h_{2}=0$ and finishes the proof of the lemma.

Remark.- After this lemma we can assume that, in our situation, $Z_{1}$ lies in both the exceptional divisor and $P$. So, for finishing our case, we are given the surface $\mathcal{S}$ defined by

$$
F=Z^{n}+\sum_{k=0}^{n-1} a_{k}(X, Y) Z^{k}
$$

and its quadratic transform $\mathcal{S}^{(1)}$ on the direction $(1: 0: 0)$, defined by

$$
F^{(1)}=Z_{1}^{n}+\sum_{k=0}^{n-1} a_{k}^{(1)}\left(X_{1}, Y_{1}\right) Z_{1}^{k},
$$

about which we know the following facts:

- The multiplicity of $\mathcal{S}$ and $\mathcal{S}^{(1)}$ is the same.

- There is a curve $P=\left(Z_{1}, X_{1}+G\left(Y_{1}\right)\right) \in \mathcal{E}_{0}\left(\mathcal{S}^{(1)}\right)$, with $\operatorname{ord}(G) \geq 2$. 
We have to prove that there is a curve $Q \in \mathcal{E}(\mathcal{S}) \backslash \mathcal{E}_{0}(\mathcal{S})$ such that $\varpi_{(1: 0: 0)}^{M}(Q)=P$. This was proved in our previous paper [5] in a characteristic-free way. In fact, the really difficult part in positive characteristic is proving that we can assume $P$ to have this particular form, and this has been done in the previous lemma.

We give a brief outline of the proof: first one shows that it is enough to prove that one can find a power series $H\left(X_{1}, Y_{1}\right)$ verifying:

- $\operatorname{ord}(H)=\operatorname{ord}(G)=\lambda>2$.

- $H$ is regular in $Y_{1}$ of order $\lambda$.

- There is a unit $u\left(X_{1}, Y_{1}\right)$ such that

$$
\frac{1}{X_{1}^{\lambda}} H\left(X_{1}, X_{1} Y_{1}\right)=u\left(X_{1}, Y_{1}\right)\left(X_{1}+G\left(Y_{1}\right)\right) .
$$

Then one proves that this power series actually exists in a constructive way (in an ample sense, of course): it consists simply in writing

$$
\begin{aligned}
& X_{1}+G\left(Y_{1}\right)=X_{1}+\sum_{i \geq \lambda} \alpha_{i} Y_{1}^{i} \\
& H\left(X_{1}, Y_{1}\right)=\sum_{k \geq \lambda}\left(\sum_{i+j=k} \beta_{i j} X_{1}^{i} Y_{1}^{j}\right) \\
& u\left(X_{1}, Y_{1}\right)=\sum_{k \geq 0}\left(\sum_{i+j=k} \gamma_{i j} X_{1}^{i} Y_{1}^{j}\right)
\end{aligned}
$$

and then imposing the third condition above. In this way it is straightforward seeing that one can, in fact, construct $H$ and $u$ with the desired properties.

\section{Case (b.1)}

There are no great differences here with respect to the null characteristic proofs. For instance, take $P=(\alpha: \beta: \gamma)$ a direction in the exceptional divisor with multiplicity $r$. Then the quadratic transform of $\mathcal{S}$ on $(\alpha: \beta: \gamma)$ has, at most, multiplicity $r$. This is plain from the very definition of multiplicity.

So the hypothesis of (b.1) are filled only if the direction chosen is one of multiplicity $n$. Eventually, changing the variables we may consider that the point is $(0: 1: 0)$ (and $\bar{F} \in K[X, Z])$. 
For proving that the quadratic transform cannot have new permitted curves note that, in (b.2), we have shown that, if a new permitted curve appears, so does the exceptional divisor (whether $\bar{F}$ is the power of a linear form or not). But $(Z, Y)$ cannot be a permitted curve, since $\overline{F^{(1)}}$ contains monomials in $K[X, Z]$ other than $Z^{n}$.

It is also clear that the quadratic transform does not erase permitted curves either. If there is a permitted curve we may take it to be $(Z, X)$, after a change of variables which does not affect $(0: 1: 0)$. Clearly this curve cannot disappear from the equimultiple locus after a quadratic transform on $(0: 1: 0)$.

This finishes the proof of the theorem.

\section{References}

[1] S.S. Abhyankar: Good points of a hypersurface. Adv. Math. 68 (1988), 87-256.

[2] H. Hironaka: Desingularization of excellent surfaces. Notes by B.M. Bennet (1967). In Resolution of Surface Singularities, Lecture Notes in Mathematics, 1101. Springer Verlag, 1984.

[3] S.B. Mulay: Equimultiplicity and hyperplanarity. Proc. Amer. Math. Soc. 89 (1983), 407-413.

[4] R. Piedra: Estudio local de singularidades de superficies sobre cuerpos de característica arbitraria. Ph. D. Thesis, Univ. de Sevilla, 1978.

[5] R. Piedra, J.M. Tornero: Equimultiple locus of embedded algebroid surfaces and blowing-up in characteristic zero. Serdica Math. J. 30 (2004) 195-206.

[6] M. Spivakovski: A counterexample to the theorem of Beppo Levi in three dimensions. Invent. Math. 96 (1989), 181-183.

[7] O. Zariski: Reduction of singularities of algebraic three dimensional varieties. Ann. of Math. 45 (1944), 472-542. 